\title{
Prognostic Factors in Patients with Malignant Pleural Mesothelioma
}

\author{
Aleksey N. Kurchenkov, MD; Vyacheslav P. Kurchin, MD, PhD, ScD; \\ Vladimir V. Zharkov, MD, PhD, ScD \\ N.N. Alexandrov National Cancer Centre of Belarus \\ Minsk, Belarus
}

\begin{abstract}
The aim of the present study was to examine the factors of prognosis in patients with malignant pleural mesothelioma (MPM) after combined and multimodality treatment, including the prognostic significance of preoperative intrapleural perfusion hyperthermo-chemotherapy (IPHC).

Material and Methods: The study included 20 patients (11 men and 9 women) aged from 30 to 70 years (mean age $51.9 \pm 8.5$ years) who underwent surgical treatment for MPM. The diagnosis of MPM was verified by immunohistochemical data. The patients were divided into two groups. Group 1 included 9 patients who underwent combined treatment that included the extrapleural pneumonectomy (EPP) and 4 courses of adjuvant chemotherapy. Group 2 included 11 patients who received multimodality treatment (IPHC, EPP, and 4 courses of adjuvant chemotherapy). All patients were followed prospectively at threemonthly intervals for the first year and six-monthly thereafter until the last time of contact or death. Statistical analysis was performed by using Kaplan-Meier method and the log-rank test. Cox-regression model was used for multivariate analysis.

Results: Patient's age over 60 years and the sarcomatoid type of the tumor can be regarded as prognostic factors for poor survival in patients with MPM who underwent EPP. Application of IPHC as a part of a multimodality treatment enhances the survivability of MPM patients.
\end{abstract}

Keywords: malignant pleural mesothelioma; extrapleural pneumonectomy; intrapleural perfusion hyperthermo-chemotherapy; multimodality treatment.

\section{Introduction}

Malignant pleural mesothelioma (MPM) is a rare disease characterized by an aggressive course and extremely poor prognosis. Tumor involves the visceral, parietal pleura, lung, pericardium, and diaphragm and metastasizes into lymph nodes and other organs. Progressive malignant pleurisy and lesion of lung parenchyma contribute to the development of cardiopulmonary failure in patients with MPM. MPM is often diagnosed in the advanced stages and $70 \%$ of patients die within a year [1].

Results of various treatments show a low efficiency. Median survival after first-line chemotherapy is 12.4 months [2]. Multimodality treatment with surgery improves median survival to 20-29 months [3-5]. Multimodality treatment is the aggressive therapy associated with high risk for elderly patients

*Corresponding author: Aleksey N. Kurchenkov, MD, researcher of the thoracic oncopathology department with the anesthesiology group of N.N. Alexandrov National Cancer Centre of Belarus Minsk,Belarus E-mail: akurchenkov@mail.ru with co-morbidities. The risk may be justified in a case of favorable prognosis. It is advisable to resort to chemotherapy or symptomatic treatment in cases of a poor prognosis with short duration of life. From this point of view adequate information about the prognostic factors affecting survival will help to select patients for multimodality treatment and in the long run increase its efficiency. Little is known about the role of preoperative intrapleural perfusion hyperthermochemotherapy (IPHC) in multimodality treatment. In this connection the aim of the present study was to examine the factors of prognosis in patients with MPM after combined and multimodality treatment, including the prognostic significance of IPHC.

\section{Material and methods}

The study included 20 patients (11 men and 9 women) aged from 30 to 70 years (mean age $51.9 \pm 8.5$ years) who underwent surgical treatment for MPM at the N.N. Alexandrov National Cancer Center of Belarus between January 2006 and December 2013 according to a randomized study on the 
comparative effectiveness of the combined and multimodality treatment of MPM. Written informed consent was obtained from each patient. The diagnosis of MPM was verified by immunohistochemical data. The patients were divided into two groups. Group 1 included 9 patients who underwent combined treatment that included the extrapleural pneumonectomy (EPP) and 4 courses of adjuvant chemotherapy. Group 2 included 11 patients who received multimodality treatment (IPHC, EPP, and 4 courses of adjuvant chemotherapy). One course of adjuvant chemotherapy included cisplatin $90 \mathrm{mg} / \mathrm{m} 2$ in 1 day, vinorelbine $30 \mathrm{mg} / \mathrm{m} 2$ in 1 and 8 days intravenously. IPHC was carried out in mode $42^{\circ} \mathrm{C}$ at ThermoChem HT- 1000 with cisplatin $120 \mathrm{mg} / \mathrm{m}^{2}$ and vinorelbine $30 \mathrm{mg} / \mathrm{m}^{2}$ for 1 hour.

All patients were followed prospectively at threemonthly intervals for the first year and six-monthly thereafter until the last time of contact or death. The follow-up review included clinical examination and assessment of chest and abdominal CT scans. The follow-up status was regularly updated in the database for each patient by a data manager. Statistical analysis was performed by using Kaplan-Meier method [6] and the log-rank test [7]. Cox-regression model was used for multivariate analysis [8]. An algorithm stepby-step "stepwise" was used to identify prognostic factors. Statistical processing of data was carried out using SPSS system version 17.0.1 for Windows.

Sex, age (under 60, and 60 and over), histological type of mesothelioma, hemoglobin, erythrocyte sedimentation rate (ESR), alanine aminotransferase, alkaline phosphatase, aspartate aminotransferase, forced expiratory volume for 1 second, history of the disease, blood loss, radical surgery (RO, R1), and treatment (IHPC or without) were studied as possible prognostic factors. The clinical characteristics of patients are shown in Table 1. Significant differences between two groups were not revealed.

Table 1.

The clinical characteristics of patients with MPM

\begin{tabular}{|l|c|c|}
\hline \multirow{2}{*}{\multicolumn{1}{|c|}{ Features }} & \multicolumn{2}{c|}{ Treatment } \\
\cline { 2 - 3 } & Group 1 & Group 2 \\
\hline Mean age (y) & $51.9 \pm 8.9$ & $51.9 \pm 9.6$ \\
\hline Gender (men/women) & $6 / 3$ & $5 / 6$ \\
\hline Tumor localization (right/left) & $5 / 4$ & $5 / 6$ \\
\hline Histology of MPM (S/E) & $1 / 8$ & $1 / 10$ \\
\hline Lymph nodes (N0/N2) & $1 / 8$ & $2 / 9$ \\
\hline Hemoglobin ( $\geq 110 /<110 \mathrm{~g} / \mathrm{L})$ & $8 / 1$ & $8 / 3$ \\
\hline ALT $(20$ IU/L/>20IU/L) & $9 / 0$ & $9 / 2$ \\
\hline AST $(20$ IU/L/>20IU/L) & $9 / 0$ & $9 / 2$ \\
\hline ALP $(120$ IU/L/>120 IU/L) & $9 / 0$ & $10 / 1$ \\
\hline FEV1 $(<1,500 \mathrm{~mL} / \geq 1,500 \mathrm{~mL})$ & $0 / 9$ & $1 / 10$ \\
\hline
\end{tabular}

$S$ - sarcomatoid type; $E$ - epithelioid type; ALP - Alkaline phosphatase

\section{Results}

TNM staging is shown in Table 2. Lymph node metastasis was found in $18(90 \%)$ of patients; it was found involving all groups of mediastinal lymph nodes on the affected side.
Stage II was diagnosed only in 1 patient. In all remaining cases, the tumor had a greater spread. The histological type of mesothelioma is shown in Table 3. Only one patient in each group had sarcomatoid type of mesothelioma.

Table 2.

Distribution of patients according to TNM staging

\begin{tabular}{|l|c|c|}
\hline \multicolumn{1}{|c|}{ TNM } & Stage & Number of patients (\%) \\
\hline T2N0M0 & II & $1(5)$ \\
\hline T3N0M0 & III & $1(5)$ \\
\hline T2N2M0 & III & $2(10)$ \\
\hline T3N2M0 & III & $16(80)$ \\
\hline \multicolumn{2}{|c|}{ All patients } & $20(100)$ \\
\hline
\end{tabular}

Table 3.

Histological type of MPM

\begin{tabular}{|l|c|}
\hline \multicolumn{1}{|c|}{ Histological type } & Number of patients (\%) \\
\hline Epithelioid MPM & $18(90)$ \\
\hline Sarcomatoid MPM & $2(10)$ \\
\hline All patients & $20(100)$ \\
\hline
\end{tabular}

As is known, IPHC has a systemic and local impact aimed at preventing a relapse of MPM. Assessment of therapeutic pathomorphism of tumor according to G.A. Lavnikova [9] was used for the analysis of the cytotoxic effect of IPHC. After IPHC, the second degree of therapeutic pathomorphism of tumor was identified. This indicates the effectiveness of IPHC in treatment of MPM.

Overall, the 5-years survival of patients after radical surgery was $23.8 \pm 12.7 \%$ (Fig. 1). Life expectancy was 29.8 months (95\% CI: 16.6-43.0 months); median survival was 18.0 months (95\% CI: 3.6-32.3 months).

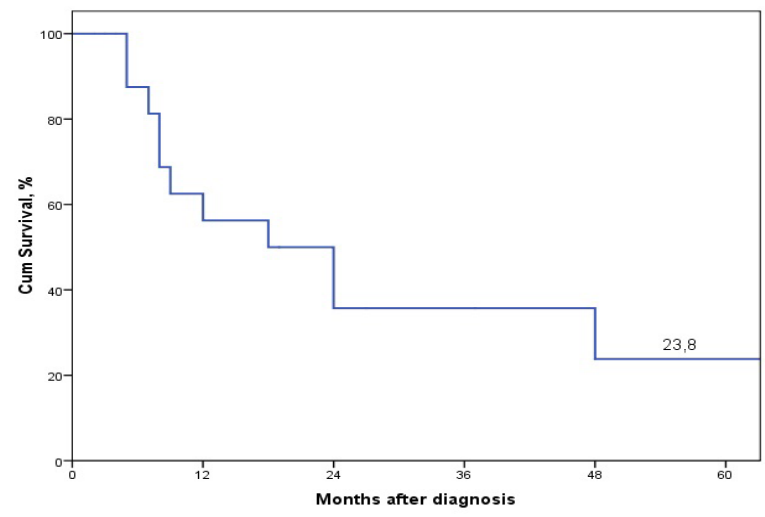

Figure 1. The 5-year survival of patients with $M P M$ after radical surgery.

Analysis of the comparative effectiveness of combined and multimodality treatment revealed that 5-year survival was statistically significantly higher when IPHC was used $(P=0.01$, Fig. 2$)$. Age, histopathological subtype and treatment were identified as independent prognostic factors according to 
multivariate analysis (Table 4). Poor prognostic factors were age $\geq 60$ years and sarcomatoid type of mesothelioma.

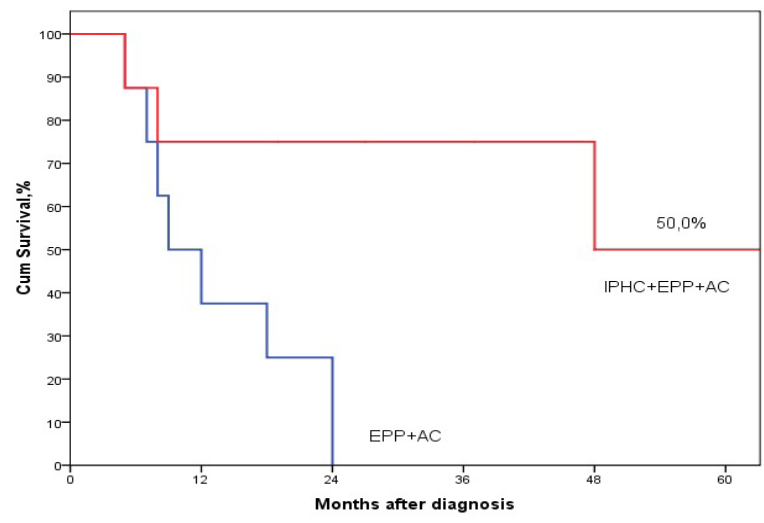

Figure 2. The 5-year survival of patients with MPM in Groups 1 and 2.

Table 4.

Prognostic factors in patients with MPM

\begin{tabular}{|c|l|c|c|c|}
\hline Variable & \multicolumn{1}{|c|}{$\begin{array}{c}\text { Evidence } \\
\text { (coding) }\end{array}$} & $\begin{array}{c}\text { Factor } \\
\beta\end{array}$ & $\begin{array}{c}\text { Level of } \\
\text { significance } \\
\mathrm{P}\end{array}$ & $\operatorname{Exp}(\beta)$ \\
\hline $\mathrm{X} 1$ & $\begin{array}{l}\text { Treatment } \\
\text { (combined - 0, } \\
\text { multimodality - 1 }\end{array}$ & -2.455 & 0.022 & 0.086 \\
\hline $\mathrm{X} 2$ & $\begin{array}{l}\text { Histological type } \\
\text { (sarcomatoid - 1, } \\
\text { epithelioid - 0) }\end{array}$ & 6.047 & 0.002 & 422,910 \\
\hline $\mathrm{X} 3$ & $\begin{array}{l}\text { Age (under 60 y - 0, } \\
60 \text { and over - 1) }\end{array}$ & 2.659 & 0.002 & 14,281 \\
\hline
\end{tabular}

The application of IPHC in multimodality treatment was found to be associated with improved survival. According to the resulting model, the hazard ratio (HR) in patients with MPM is defined by the equation:

$$
\begin{aligned}
& \mathrm{HR}=\frac{\exp \left(-2.44 X_{1}^{*}+6.047 X_{2}^{*}+2.659 X_{3}^{*}\right)}{\exp \left(-2.44 X_{1}+6.047 X_{2}+2.659 X_{3}\right)}=\exp [-2.44 \bullet \\
& \left.\left(X_{1}^{*}-X_{1}\right)+6.047\left(X_{2}^{*}-X_{2}\right)+2.659\left(X_{3}^{*}-X_{3}\right)\right]
\end{aligned}
$$

where $X_{1}^{*}, X_{2}^{*}, X_{3}^{*}$ and $X_{1}, X_{2}, X_{3}$ are values of prognostic variables in 2 patients.

Poor prognosis for survival occurs in patients over 60 years of age with the sarcomatoid type of mesothelioma and without IPHC that corresponds to a set of prognostic variables:

$$
X_{1}^{*}=0, X_{2}^{*}=1 \text {, and } X_{3}^{*}=1
$$
variables:

In a favorable prognosis, the value of the prognostic

$$
X_{1}=1, X_{2}=0 \text {, and } X_{3}=0
$$

The value of HR for patients with poor and favorable prognosis will be:

$\mathrm{HR}=\exp [-2.455(0-1)+6.047(1-0)+2.659(1-0)]=\exp (11.161)=70,333.3$
In this way, the conditional probability of death per unit of time will be in 70,333.3 times higher in patients with poor prognosis compared to those with a favorable prognosis. For patients over 60 years of age with epithelioid mesothelioma and treatment without IPHC, the conditional probability of death will be in 166.3 times higher compared to patients under 60 years of age who will receive the multimodality treatment with IPHC:

$\mathrm{HR}=\exp [-2.455(0-1)+6.047(0-0)+2.659(1-0)]=\exp (11.146)=166.3$

If patients differ only in the treatment used, HR will be:

$\mathrm{HR}=\exp [-2.455(0-1)+6.047(0)+2.659(0)]=\exp (2.455)=11.7$

This confirms the favorable prognosis for IPHC application for treatment of mesothelioma.

Figure 3 demonstrates the influence of prognostic factors on the survival rates in patients with MPM. In the presence of unfavorable factors all patients died within a year. With favorable prognostic factors, the 5-year survival was $57.1 \pm 24.9 \%$. The actual data are in good agreement with the survival estimated according to the Cox model.

\section{Discussion}

There is relatively little research on the survival predictors in patients with MPM who have had the surgical and multimodal treatment. D.J. Sugarbaker et al. analyzed 183 patients and concluded that for the non-epithelioid cell type of MPM, positive resection margins and metastatic extrapleural nodes were negative prognostic factors [10].

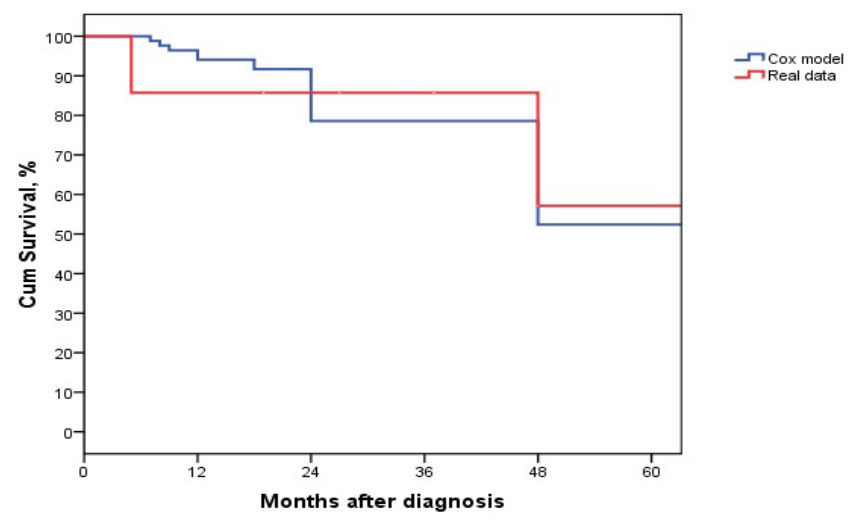

Figure 3. The estimated survival for patients with MPM according to the Cox model

R.M. Flores et al. have shown the benefit of multimodality therapy. Other positive factors according to this research were gender (female), left-side tumor, lack of pain syndrome and lack of exposure to asbestos [11].

M. de Perrot et al. examined the results of trimodality therapy with cisplatin-based chemotherapy followed by EPP and adjuvant high-dose (50 to $60 \mathrm{~Gy}$ ) hemithoracic radiation therapy for MPM. According to this study, the presence of N2 disease was a significant marker of poor outcome, despite completion of the entire trimodality regimen. [12]. 
According to the largest international database (3101 patients with MPM from 15 centers and 4 continents) developed by the International Association for the Study of Lung Cancer Staging Committee, median survivals by clinical TNM and pathological TNM were similar: stage I, 21 months; stage II, 19 months; stage III, 16 months; and stage IV, 12 months. Median survival by histology: epithelioid 19 months, biphasic 13 months, and sarcomatoid 8 months. By multivariable analyses, significant differences in overall survival were seen for: T4 versus T3 and T3 versus T2 but not $\mathrm{T} 2$ versus $\mathrm{T} 1$; $\mathrm{N} 0$ versus $\mathrm{N} 1$ and $\mathrm{N} 2$ but not N1 versus N2; stages III and IV versus I but not II versus I; epithelioid histology versus other; age of female versus age of male; and palliative versus curative-intent surgery [13].

A Report from the IASLC Staging Committee analyzed prognostic variables in a surgical population, which are supplementary to previously published CORE variables (stage, histology, sex, age, and type of procedure). Lack of adjuvant therapy, along with the presence of asbestos exposure, weight loss, and chest pain, as well as low hemoglobin, high platelet count, and high white blood count, was found to be associated with a worse prognosis independent of the CORE variables [14].

Our data suggest the prognostic role of MPM histology and age in patients managed with EPP, which is consistent with other studies. In our study, the predictive value of IPHC with MPM has been shown for the first time. The use of IPHC in multimodality treatment enhances the survival rate in MPM patients; this fact confirms the synergetic antitumor effect for the combination of the hyperthermia with chemotherapy against the neoplastic cells [15]. Unfortunately, the small number of patients restricts the possibility for evaluating other prognostic factors. However, our results showed an improvement in survival for MPM patients under 60 years of age, who were managed with IPHC and had the epithelioid tumor type.

\section{Conclusion}

Patient's age over 60 years and the sarcomatoid type of the tumor can be regarded as prognostic factors for poor survival in patients with MPM who underwent EPP. Application of IPHC as a part of a multimodality treatment enhances the survivability of MPM patients.

\section{Competing interests}

The authors declare that they have no competing interests.

\section{References}

1. Milano M T, Zhang H. Malignant pleural mesothelioma: a population-based study of survival. J Thorac Oncol 2010; 5(11):1841-8.

2. Vogelzang N J, Rusthoven J J, Symanowski J, Denham C, Kaukel E, Ruffie P, et al. Phase III study of pemetrexed in combination with cisplatin versus cisplatin alone in patients with malignant pleural mesothelioma. J Clin Oncol 2003;
21(14): 2636-44.

3. Yan TD, Boyer M, Tin M, McLean J, Banon P G, McCaughan BC. Treatment failure after extrapleural pneumonectomy for malignant pleural mesothelioma. J Thorac Dis 2009; 1(1):23-8.

4. Krug LM, Pass HI, Rusch VW, Kindler HL, Sugarbaker DJ, Rosenzweig KE, et al. Multicenter phase II trial of neoadjuvant pemetrexed plus cisplatin followed by extrapleural pneumonectomy and radiation for malignant pleural mesothelioma. J Clin Oncol 2009; 27(18):3007-13.

5. Weder W, Opitz I. Multimodality therapy for malignant pleural mesothelioma. Ann Cardiothorac Surg 2012; 1(4):5027.

6. Kaplan E L, Meier P. Nonparametric estimation from incomplete observations. J Am Stat Ass 1958; 53:457-81.

7. Mantel N. Evaluation of survival data and two new rank order statistics arising in its consideration. Cancer Chemother Rep 1966; 50(3):163-70.

8. Cox D R. Regression models and life tables. J R Stat Soc (B) $1972 ; 34: 187-229$.

9. Lavnikova GA. Some regularities of radiation pathomorphism of human tumors and their practical use. Bull Acad Med Sci 1976; 6:13-9.[Article in Russian].

10. Sugarbaker DJ, Flores RM, Jaklitsch MT, Richards WG, Strauss GM, Corson JM, et al. Resection margins, extrapleural nodal status, and cell type determine postoperative longterm survival in trimodality therapy of malignant pleural mesothelioma: results in 183 patients. J Thorac Cardiovasc Surg 1999; 117(1): 54-63.

11. Flores RM, Pass HI, Seshan VE, Dycoco J, Zakowski M, Carbone M, et al. Extrapleural pneumonectomy versus pleurectomy/decortication in the surgical management of malignant pleural mesothelioma: results in 663 patients. J Thorac Cardiovasc Surg 2008; 135(3): 620-6.

12. de Perrot M, Feld R, Cho BC, Bezjak A, Anraku M, Burkes $\mathrm{R}$, et al. Trimodality therapy with induction chemotherapy followed by extrapleural pneumonectomy and adjuvant high-dose hemithoracic radiation for malignant pleural mesothelioma. J Clin Oncol 2009; 27(9):1413-8.

13. Rusch VW, Giroux D, Kennedy C, Ruffini E, Canqir AK, Rice D, et al. Initial analysis of the International Association for the Study of Lung Cancer mesothelioma database. J Thorac Oncol 2012; 7(11):1631-9.

14. Pass HI, Giroux D, Kennedy C, Ruffini E, Canqir AK, Rice D, et al. Supplementary prognostic variables for pleural mesothelioma: A report from the IASLC Staging Committee. J Thorac Oncol 2014; 9(6):856-64.

15. Koga S, Hamazoe R, Maeta M, Shimizu N, Kanayama H, Osaki Y. Treatment of implanted peritoneal cancer in rats by continuous hyperthermic peritoneal perfusion in combination with an anticancer drug. Cancer Res 1984; 44(5):1840-2. 